\title{
ON LINEAR CONTINUOUS OPERATORS BETWEEN DISTINGUISHED SPACES $C_{p}(X)$
}

\author{
JERZY KA̧KOL AND ARKADY LEIDERMAN
}

\begin{abstract}
As proved in [16, for a Tychonoff space $X$, a locally convex space $C_{p}(X)$ is distinguished if and only if $X$ is a $\Delta$-space. If there exists a linear continuous surjective mapping $T: C_{p}(X) \rightarrow C_{p}(Y)$ and $C_{p}(X)$ is distinguished, then $C_{p}(Y)$ also is distinguished [17.

Firstly, in this paper we explore the following question: Under which conditions the operator $T: C_{p}(X) \rightarrow C_{p}(Y)$ above is open? Secondly, we devote a special attention to concrete distinguished spaces $C_{p}([1, \alpha])$, where $\alpha$ is a countable ordinal number. A complete characterization of all $Y$ which admit a linear continuous surjective mapping $T: C_{p}([1, \alpha]) \rightarrow C_{p}(Y)$ is given. We also observe that for every countable ordinal $\alpha$ all closed linear subspaces of $C_{p}([1, \alpha])$ are distinguished, thereby answering an open question posed in [17.

Using some properties of $\Delta$-spaces we prove that a linear continuous surjection $T: C_{p}(X) \rightarrow C_{k}(X)_{w}$, where $C_{k}(X)_{w}$ denotes the Banach space $C(X)$ endowed with its weak topology, does not exist for every infinite metrizable compact $C$-space $X$ (in particular, for every infinite compact $X \subset \mathbb{R}^{n}$ ).
\end{abstract}

\section{INTRODUCTION}

A locally convex space (lcs) $E$ is called distinguished if its strong dual $E_{\beta}^{\prime}=$ $\left(E^{\prime}, \beta\left(E^{\prime}, E\right)\right)$ is a barrelled space. A. Grothendieck [11] proved that a metrizable lcs is distinguished if and only if $E_{\beta}^{\prime}$ is bornological. Also, if all bounded subsets of the strong dual $E_{\beta}^{\prime}$ of a metrizable lcs are metrizable, then $E$ is distinguished [11]. Recall that the strong topology $\beta$ on $E^{\prime}$ is the topology of uniform convergence on bounded subsets of $E$. A subset $A \subset E$ is bounded if is absorbed by each neighbourhood of zero in $E$. We refer the reader to the survey article of K. D. Bierstedt and J. Bonet [7], where many classes of distinguished Fréchet lcs are presented (reflexive spaces, Montel spaces, nuclear spaces, Schwartz spaces, etc).

Date: July 13, 2021.

2010 Mathematics Subject Classification. Primary 54C35; Secondary 46A03, 46A20.

Key words and phrases. Distinguished locally convex space, linear continuous operator, $\Delta$ space, countable ordinal.

The first named author is supported by the GAČR project 20-22230L and RVO: 67985840 . The authors thank J.C. Ferrando for providing a short argument in Remark 2.5)(iii) and W.B. Johnson and T. Kania for a very helpful advice and discussion about Theorem 3.2 
In this paper we continue the study of distinguished lcs in the frame of spaces $C_{p}(X)$ and $C_{k}(X)$ (developed earlier in [8], [9], [10], [16], [17]). By $C_{p}(X)$ and $C_{k}(X)$ we mean the spaces of all real-valued continuous functions on a Tychonoff space $X$ endowed with the topology of pointwise convergence $\tau_{p}$ and the compactopen topology $\tau_{k}$, respectively.

Recall that for a vector space $E$ the finest locally convex topology $\xi$ of $E$ is generated by the family of all absolutely convex and absorbing subsets of $E$ which form a base of neighbourhoods of zero for the topology $\xi$. We denote by $\mathbb{N}$ the infinite countable discrete space. It is obvious that $C_{p}(\mathbb{N})=\mathbb{R}^{\mathbb{N}}$ is the only distinguished Fréchet lcs $E$ for which its strong dual carries the finest locally convex topology. In fact, $C_{p}(\mathbb{N})_{\beta}^{\prime}$ is isomorphic to $\varphi$, i.e. the $\aleph_{0}$-dimensional vector space with the finest locally convex topology. On the other hand, one can characterize distinguished lcs $C_{p}(X)$ as follows: $C_{p}(X)$ is distinguished if and only if $C_{p}(X)_{\beta}^{\prime}$ carries the finest locally convex topology, see [8], [9].

We proved that $C_{p}(X)$ is distinguished if and only if $X$ is a $\Delta$-space [16].1. The class $\Delta$ of $\Delta$-spaces naturally extends the class of $\Delta$-sets of reals. Note that the original definition of a $\Delta$-set $A \subset \mathbb{R}$ is due to G. Reed and E. van Douwen [28].

A topological space $X$ is said to be a $\Delta$-space if for every decreasing sequence $\left\{D_{n}: n \in \omega\right\}$ of subsets of $X$ with empty intersection, there is a decreasing sequence $\left\{V_{n}: n \in \omega\right\}$ consisting of open subsets of $X$, also with empty intersection, and such that $D_{n} \subset V_{n}$ for every $n \in \omega[16]$.

Quite recently a range of new facts about $\Delta$-spaces has been obtained by the authors. We proved that every Čech-complete (in particular, compact) $X \in \Delta$ is scattered [16]. Moreover, every countably compact space is scattered [17]. Class $\Delta$ contains all separable compact spaces of the Isbell-Mrówka type, while there are compact scattered spaces $X \notin \Delta$, for example, ordinal space [0, $\left.\omega_{1}\right]$ [16]. In fact, every compact $\Delta$-space must have countable tightness [17]. Although the property of being a $\Delta$-space is not inherited by continuous images, the class $\Delta$ does preserve closed continuous images [16]. Furthermore, a countable union of compact $\Delta$-spaces is also a $\Delta$-space; in particular, $\sigma$-product of any family consisting of scattered Eberlein compact spaces is a $\Delta$-space [17]. The next result is a matter of high importance to us.

Theorem 1.1. [17] The class $\Delta$ is invariant under the relation of l-dominance, i.e. if $C_{p}(X)$ is distinguished and there exists a linear continuous surjective mapping $T: C_{p}(X) \rightarrow C_{p}(Y)$, then $C_{p}(Y)$ also is distinguished.

The focus in this paper is on the following question: Under which conditions the operator $T: C_{p}(X) \rightarrow C_{p}(Y)$ above is open? We notice that in general there are plenty of linear continuous non-open surjections between $C_{p}(X)$-spaces.

\footnotetext{
${ }^{1}$ We should mention that independently and simultaneously an analogous description of distinguished $C_{p}$-spaces (but formulated in different terms) appeared in [10].
} 
Proposition 1.2. 25] Let $X$ be a Tychonoff space and $h: X \rightarrow X$ be a continuous mapping such that the orbit of at least one point is infinite. Then the mapping $T: C_{p}(X) \rightarrow C_{p}(X)$ defined by $T f(x)=\lambda f(x)+f(h(x))$ is a linear continuous non-open surjection for each $\lambda$ with $|\lambda|>1$.

In Section 2 we prove the following open mapping theorem for spaces $C_{p}(X)$ over $\Delta$-spaces $X$.

Theorem 1.3. Let $X$ be a $\Delta$-space. Consider the following assertions for a linear continuous surjection $T: C_{p}(X) \rightarrow C_{p}(Y)$.

(1) $C_{p}(X)$ admits a base $\mathcal{U}$ of absolutely convex neighbourhoods of zero such that $\operatorname{cl} T(U) \subset 2 T(U)$ for each $U \in \mathcal{U}$.

(2) $T$ is an open mapping.

(3) $C_{p}(X)$ admits a base $\mathcal{U}$ of absolutely convex closed neighbourhoods of zero such that $T(U)$ is closed for each $U \in \mathcal{U}$.

Then (3) $\Rightarrow$ (2) $\Leftrightarrow$ (1).

Corollary 1.4. If $X$ and $Y$ are $\Delta$-spaces and $T: C_{p}(X) \rightarrow C_{p}(Y)$ is a linear continuous bijection, then $T$ is an isomorphism if and only if any condition from Theorem 1.3 holds.

It is easy to see that in above Proposition [1.2, $X$ might be selected as a $\Delta$ space for which a continuous linear non-open surjection $T: C_{p}(X) \rightarrow C_{p}(X)$ exists but $T$ lacks condition (3) of Theorem 1.3. This happens, for example, if $C_{p}(X)$ is barrelled. Clearly, there exist Banach spaces $E$ and $F$ and linear continuous surjections $T: E \rightarrow F$ with non-closed $T(S)$ for the closed unit ball $S \subset E$; injective linear continuous maps $T: E \rightarrow F$ such that $T(S)$ is closed were studied under the name semi-embedding, see [26].

We stress that our proof of Theorem 1.3 rely inevitably on the assumption that $X$ is a $\Delta$-space. Recall that the unit segment $[0,1]$ is not a $\Delta$-space. A challenging problem of whether a non-open continuous linear surjection $T: C_{p}[0,1] \rightarrow$ $C_{p}[0,1]$ satisfying condition (3) of Theorem 1.3 exists, remains unsolved.

We devote a special attention to $\operatorname{lcs} C_{p}(X)$ for some concrete $\Delta$-spaces $X$. For any ordinal number $\alpha$ let $[1, \alpha]$ denote the ordered compact space consisting of all ordinals $\gamma \leq \alpha$. The symbols $\omega$ and $\omega_{1}$ stand for the first infinite and the first uncountable ordinal numbers, respectively. S. Gul'ko showed that for any pair of infinite countable ordinals $\alpha$ and $\beta$, the spaces $C_{p}([1, \alpha])$ and $C_{p}([1, \beta])$ are uniformly homeomorphic, see [12]. Theorem 3.2 shows however that if $\alpha \leq$ $\beta<\omega_{1}$ and the spaces $C_{p}([1, \alpha])$ and $C_{p}([1, \beta])$ are not linearly isomorphic, then $C_{p}([1, \beta])$ is not a continuous linear image of $C_{p}([1, \alpha])$. Thus our results are related to the research area of linear topological classification of function spaces originated long time ago in the pioneering work of C. Bessaga and A. Pełczyński [6]. A complete linear topological classification of the spaces $C_{p}([1, \alpha])$ has been obtained independently in [13] (for all $\alpha$ ) and [3] (for countable $\alpha$ ). 
We also observe that for every countable ordinal compact space $X=[1, \alpha]$ the space $C_{p}(X)$ is hereditarily distinguished in the following sense: all closed linear subspaces of $C_{p}(X)$ are distinguished (Theorem 3.4). This result answers in the affirmative Problem 2.18 posed in [17].

The argument used in the proof of Theorem 1.3 applies to provide a "linear version" of M. Krupski and W. Marciszewski results from [20], [22] about the non-existence of a homeomorphism between spaces $C_{p}(X)$ and $C_{k}(Y)_{w}$ over certain compact spaces $X$ and $Y$, where $C_{k}(Y)_{w}$ denotes the Banach space $C(Y)$ endowed with its weak topology $w$. Although we do not know if there exists an infinite compact space $X$ admitting a linear continuous surjection $T: C_{p}(X) \rightarrow C_{k}(X)_{w}$, we show (Theorem 4.4) that any infinite metrizable compact $C$-space $X$, (in particular, any infinite metrizable finite-dimensional compact space $X$ ), does not admit such a mapping.

Our notations are standard, the reader is advised to consult with the monograph [14] and the survey paper [30] for the notions which are not explicitly defined in the text. In the article we pose several open questions.

\section{Proof of Theorem 1.3 and $\varphi$-Dual subspaces}

A lcs $E$ is called free if $E$ carries the finest locally convex topology. Each free lcs has only finite-dimensional bounded sets, see for example [5, Theorem 2.1]. On the other hand, for every Tychonoff $X$, in the space $C_{p}(X)_{\beta}^{\prime}$ all bounded sets are finite-dimensional, but $C_{p}(X)_{\beta}^{\prime}$ is not necessarily free. For the proofs of the next characterizations we refer to [9] and [16].

Theorem 2.1. Let $X$ be a Tychonoff space.

(a) [8], 9] $C_{p}(X)$ is distinguished if and only if $C_{p}(X)_{\beta}^{\prime}$ is free.

(b) [9] $C_{p}(X)$ is distinguished if and only if for each bounded set $A \subset \mathbb{R}^{X}$ there exists a bounded set $B \subset C_{p}(X)$ such that $A \subset c l_{\mathbb{R}^{X}}(B)$.

(c) [16] $C_{p}(X)$ is distinguished if and only if $X$ is a $\Delta$-space.

We start with the following theorem describing the free spaces $C_{k}(X)_{\beta}^{\prime}$ which (together with Corollary 2.3) will be used in proving Theorem 1.3. By $C_{p}(X)^{\prime}$ and $C_{k}(X)^{\prime}$ we denote the topological dual of $C_{p}(X)$ and $C_{k}(X)$, respectively.

Theorem 2.2. Let $X$ be a Tychonoff space. Let $\vartheta_{X}$ be the strong topology of $C_{k}(X)_{\beta}^{\prime}$. Then the following assertions are equivalent:

(1) The topology $\vartheta_{X}$ is the finest locally convex topology of $C_{k}(X)_{\beta}^{\prime}$.

(2) $\tau_{k}=\tau_{p}$ and $C_{p}(X)$ is distinguished.

(3) For each bounded set $A \subset \mathbb{R}^{X}$ there exists a bounded set $B$ in $C_{k}(X)$ such that $A \subset c l_{\mathbb{R}^{X}}(B)$.

Proof. Implications $(2) \Rightarrow(1)$ and $(2) \Rightarrow(3)$ are straightforward consequences of Theorem [2.1. $(1) \Rightarrow(2)$ : Note that the topology $\tau_{k}$ on $C(X)$ is $\tau_{p}$-polar, i.e. $\tau_{k}$ 
admits a base of absolutely convex neighbourhoods of zero consisting of $\tau_{p}$-closed sets. Hence, if $S$ is a $\tau_{k}$-bounded set in $C_{k}(X)$, then the closure $c l_{\tau_{p}}(S)$ is still $\tau_{k}$-bounded.

Assume that (1) holds. Since $\tau_{p} \leq \tau_{k}$, the strong topology $\beta_{X}$ of $C_{p}(X)^{\prime}$ is finer than the strong topology $\vartheta_{X}$ of $C_{k}(X)^{\prime}$ restricted to $C_{p}(X)^{\prime}$ (shortly $\left.\vartheta_{X} \mid C_{p}(X)^{\prime} \leq \beta_{X}\right)$. As the topology $\vartheta_{X}$ is the finest locally convex topology on $C_{k}(X)^{\prime}$, we have in fact the equality $\vartheta_{X} \mid C_{p}(X)^{\prime}=\beta_{X}$. Hence $\left(C_{p}(X)^{\prime}, \beta_{X}\right)$ is a topological subspace of $\left(C_{k}(X)^{\prime}, \vartheta_{X}\right)$ and carries the finest locally convex topology, so $C_{p}(X)$ is distinguished by Theorem 2.1. Next we show that $\tau_{p}=\tau_{k}$.

We know that for every absolutely convex neighbourhood of zero $U$ in the space $\left(C_{p}(X)^{\prime}, \beta_{X}\right)$ there exists an absolutely convex neighbourhood of zero $V$ in $\left(C_{k}(X)^{\prime}, \vartheta_{X}\right)$ such that $V \cap C_{p}(X)^{\prime} \subset U$. Consequently, for each $\tau_{p}$-closed bounded absolutely convex set $B \subset C_{p}(X)$ there exists an absolutely convex $\tau_{k}$-bounded set $S \subset C_{k}(X)$ with $S^{\bullet} \cap C_{p}(X)^{\prime} \subset B^{\circ}$, where

$$
\begin{aligned}
& S^{\bullet}=\left\{x^{*} \in C_{k}(X)^{\prime}:\left|x^{*}(f)\right| \leq 1, f \in S\right\}, \\
& B^{\circ}=\left\{x^{*} \in C_{p}(X)^{\prime}:\left|x^{*}(f)\right| \leq 1, f \in B\right\} .
\end{aligned}
$$

Hence, $\left\{x^{*} \in C_{p}(X)^{\prime}:\left|x^{*}(f)\right| \leq 1, f \in c l_{\tau_{p}}(S)\right\} \subset\left\{x^{*} \in C_{p}(X)^{\prime}:\left|x^{*}(f)\right| \leq\right.$ $1, f \in S\} \subset\left\{x^{*} \in C_{p}(X)^{\prime}:\left|x^{*}(f)\right| \leq 1, f \in B\right\}$, what shows that the polar set $B^{\circ \circ}=\left\{x^{*} \in C_{p}(X)^{\prime}:\left|x^{*}(f)\right| \leq 1, f \in B\right\}^{\circ}$ is contained in the polar set $\left(c l_{\tau_{p}}(S)\right)^{\circ \circ}=\left\{x^{*} \in C_{p}(X)^{\prime}:\left|x^{*}(f)\right| \leq 1, f \in c l_{\tau_{p}}(S)\right\}^{\circ}$, where the corresponding polars are taken in the dual pair $\left(C_{p}(X)^{\prime}, C_{p}(X)\right)$.

By the bipolar theorem [14, Theorem 8.2.2], B= $B^{\circ \circ} \subset\left(c_{\tau_{p}}(S)\right)^{\circ \circ}=c l_{\tau_{p}}(S)$. Since $c l_{\tau_{p}}(S)$ is $\tau_{k}$-bounded, every $\tau_{p}$-bounded set in $C(X)$ is $\tau_{k}$-bounded. On the other hand, $C_{p}(X)$ is quasibarrelled, i.e. every absolutely convex closed set absorbing bounded sets in $C_{p}(X)$ is a neighbourhood of zero, see [14, 11.7.3]. Now take a $\tau_{p}$-closed absolutely convex neighbourhood of zero $U$ in $\tau_{k}$. Since, as we proved, every $\tau_{p}$-bounded set is $\tau_{k}$-bounded, the set $U$ absorbs bounded sets in $C_{p}(X)$. Hence, $U$ is also a neighbourhood of zero in $\tau_{p}$, so $\tau_{p}=\tau_{k}$.

$(3) \Rightarrow(2)$ : Take a bounded set $A \subset C_{p}(X)$. Then there exists a bounded set $B \subset C_{k}(X)$ with $A \subset c l_{\mathbb{R}^{X}}(B) \cap C_{p}(X)\left(\subset c l_{\tau_{p}}(B)\right)$. Clearly, the closure $c l_{\tau_{p}}(B)$ is still $\tau_{k}$-bounded. Therefore, the topologies $\tau_{p}$ and $\tau_{k}$ have the same bounded sets, and then $\tau_{p}=\tau_{k}$. Applying Theorem 2.1 we deduce that $C_{p}(X)$ is distinguished.

A similar argument, as used in the proof of Theorem 2.2 ((1) $\Rightarrow(2))$ for the case $\xi=\tau_{k}$, applies for the next Corollary 2.3. Analogously, one shows that bounded sets in both topologies $\xi$ and $\tau_{p}$ are the same. Since $C_{p}(X)$ is quasibarrelled, $\tau_{p}$-polarity of $\xi$ yields the equality $\xi=\tau_{p}$.

Corollary 2.3. Let $\xi \geq \tau_{p}$ be a locally convex topology on $C(X)$ which is $\tau_{p^{-}}$ polar, i.e. $\xi$ has a base of absolutely convex $\tau_{p}$-closed neighbourhoods of zero. If $(C(X), \xi)_{\beta}^{\prime}$ is free, then $\xi=\tau_{p}$. 
Example 2.4. If $X$ is any uncountable compact space, then the Banach space $C_{k}(X)$ is evidently distinguished but $C_{k}(X)_{\beta}^{\prime}$ is not free.

Proof of Theorem 1.3. (3) $\Rightarrow(2)$ : Let $Q: C_{p}(X) \rightarrow C_{p}(X) / \operatorname{ker}(T)$ be the quotient map and let $\hat{T}: C_{p}(X) / \operatorname{ker}(T) \rightarrow C_{p}(Y)$ be a map associated with $T$. It is well known that the adjoint map $Q^{*}:\left(C_{p}(X) / \operatorname{ker}(T)\right)_{\beta}^{\prime} \rightarrow C_{p}(X)_{\beta}^{\prime}$ is continuous and injective. Since $C_{p}(X)_{\beta}^{\prime}$ is free, the space $\left(C_{p}(X) / \operatorname{ker}(T)\right)_{\beta}^{\prime}$ is free, too. Moreover, $\widehat{T}$ is injective and $\widehat{T}(Q(U))=\widehat{T}(\operatorname{cl}(Q(U)))=T(U)$ is closed in $C_{p}(Y)$ for $U \in \mathcal{U}$. Hence there exists a locally convex topology $\xi$ on $C(Y)$ which is $\tau_{p}$-polar with $\tau_{p} \leq \xi$ and $\widehat{T}: C_{p}(X) / \operatorname{ker}(T) \rightarrow(C(Y), \xi)$ is an isomorphism. Since $(C(Y), \xi)_{\beta}^{\prime}$ is free, Corollary 2.3 applies to get that $\tau_{p}=\xi$, so $T$ is open.

$(2) \Rightarrow(1)$ is clear. $(1) \Rightarrow(2)$ : First note that to get the conclusion of Corollary 2.3 it is enough to assume that $\xi$ has a base $\mathcal{U}$ of absolutely convex neighbourhoods of zero with $c l_{\tau_{p}}(U) \subset 2 U$ for each $U \in \mathcal{U}$. This assumption applies to show that $c l_{\tau_{p}}(B)$ is $\xi$-bounded for each $\xi$-bounded $B \subset C(X)$, which is the essential part used in the proof of Theorem 2.2. Next, we argue as in the proof $(3) \Rightarrow(2)$ above.

Remark 2.5. (i) Theorem 1.3 fails if $C_{p}(X)$ is replaced by $C_{k}(X)$. Indeed, for every infinite compact space $X$, if $T: C_{k}(X) \rightarrow C_{p}(X)$ is the identity map, then $T$ satisfies condition (3) but $T$ is not open.

(ii) If $C_{p}(Y)$ is a barrelled lcs, i.e. every closed absolutely convex absorbing set in $C_{p}(Y)$ is a neighbourhood of zero, (see [14, Corollary 11.7.6]), then the implication $(3) \Rightarrow(2)$ of Theorem 1.3 holds for each $C_{p}(X)$.

(iii) The most natural situation when a linear continuous surjective mapping $T: C_{p}(X) \rightarrow C_{p}(Y)$ both is open and satisfies condition (3) of Theorem 1.3 is the following: $X$ is normal, $Y$ is a closed subspace of $X$ and $T:\left.f \mapsto f\right|_{Y}$ is the restriction map. We do not need to assume that $C_{p}(X)$ is distinguished. For any finite set $A \subset X$ and a real number $r>0$ denote $U_{A, r}=\{f \in C(X):|f(x)| \leq$ $r$ for every $x \in A\}$. Define the family of sets $\mathcal{U}$ consisting of all sets $U_{A, r}$ of such a form. Then $\mathcal{U}$ is a base of absolutely convex closed neighbourhoods of zero in $C_{p}(X)$. We claim that $T\left(U_{A, r}\right)$ is closed in $C_{p}(Y)$ for every $U_{A, r} \in \mathcal{U}$. Indeed, let $B=A \cap Y$, then applying the Tietze-Urysohn lemma, it is easy to see that $T\left(U_{A, r}\right)=\{f \in C(Y):|f(x)| \leq r$ for every $x \in B\}$. It follows that $T$ satisfies condition (3).

We complete this section with a theorem illustrating a role of distinguished spaces $C_{p}([1, \omega])$ and $C_{p}(\mathbb{N})$. In what follows, if lcs $E_{1}$ are $E_{2}$ are isomorphic, we write $E_{1} \approx E_{2}$. A vector subspace $F$ of $E$ will be called $\varphi$-dual if $F_{\beta}^{\prime} \approx \varphi$.

Proposition 2.6. Let $F \subset C_{p}(X)$ be a vector subspace endowed with the topology induced from $C_{p}(X)$. If $F$ is $\varphi$-dual, then $F$ is a metrizable and separable space. 
Proof. By our assumptions, the strong dual $F_{\beta}^{\prime}$ of $F$ is isomorphic to $\varphi$. The strong bidual $F_{\beta}^{\prime \prime}$ of $F$ is isomorphic to $\mathbb{R}^{\omega}$, which does not admit a weaker Hausdorff vector topology, see [27, Corollary 2.6.5]. Hence the weak*-topology $\sigma\left(F^{\prime \prime}, F^{\prime}\right)$ on $F^{\prime \prime}$ coincides with the original strong topology of $F_{\beta}^{\prime \prime}$. Consequently, $\left(F^{\prime \prime}, \sigma\left(F^{\prime \prime}, F^{\prime}\right)\right.$ ) (so also $\supset\left(F, \sigma\left(F, F^{\prime}\right)\right)$ ) is metrizable and separable. Since the original topology $\tau_{p}$ of $C_{p}(X)$ is the weak topology, we deduce that $\tau_{p} \mid F=$ $\sigma\left(F, F^{\prime}\right)$ is metrizable and separable.

Theorem 2.7. The following assertions are equivalent:

(1) $C_{p}(X)$ contains a $\varphi$-dual subspace complemented in $C_{p}(X)$.

(2) Either $C_{p}([1, \omega])$ or $C_{p}(\mathbb{N})$ is a continuous linear image of $C_{p}(X)$.

Proof. $(1) \Rightarrow(2): C_{p}(X)$ contains some complemented subspace $F$ with $F_{\beta}^{\prime} \approx \varphi$.

Case 1. $X$ is pseudocompact, i.e. every $f \in C(X)$ is bounded on $X$. We know that $F$ is a metrizable and separable space, by Proposition 2.6. In this situation we can apply [4, Theorem 1] (implication $(5) \Rightarrow(4)$ ) and conclude that there exists a continuous linear map from $C_{p}(X)$ onto $\left(c_{0}\right)_{p}$, where $\left(c_{0}\right)_{p}=\left\{\left(x_{n}\right)_{n \in \omega} \in\right.$ $\left.\mathbb{R}^{\omega}: x_{n} \rightarrow 0\right\}$, and $\left(c_{0}\right)_{p} \approx C_{p}([1, \omega])$.

Case 2. $X$ is not pseudocompact. By [2, Section 4], $C_{p}(X)$ contains a complemented copy of $C_{p}(\mathbb{N})$, so $C_{p}(X)$ can be mapped onto $C_{p}(\mathbb{N})$.

$(2) \Rightarrow(1)$ : Assume that $T: C_{p}(X) \rightarrow C_{p}(\mathbb{N})$ is a continuous linear surjection. If every $g \in C(X)$ is bounded, then $C_{p}(X)=\bigcup_{n \in \omega}\{g \in C(X):|g(x)| \leq n\}$. Hence $T\left(C_{p}(X)\right)$ would be covered by a sequence of bounded sets, which is impossible by the Baire Category Theorem. Therefore $C_{p}(X)$ contains a complemented copy of $\mathbb{R}^{\omega} \approx C_{p}(\mathbb{N})$, by [2, Section 4]. Finally, assume that $C_{p}([1, \omega])$ is a continuous linear image of $C_{p}(X)$. By [4, Theorem 1] (implication $(4) \Rightarrow(2)$ ), $C_{p}(X)$ contains a complemented copy of $\left(c_{0}\right)_{p} \approx C_{p}([1, \omega])$. In all cases $C_{p}(\mathbb{N})$ or $C_{p}([1, \omega])$ are $\varphi$-dual subspaces of $C_{p}(X)$.

Corollary 2.8. For infinite Tychonoff spaces $X$ and $Y$ the lcs $C_{p}(X \times Y)$ always contains a complemented $\varphi$-dual subspace.

Proof. If $X \times Y$ is not pseudocompact, then $C_{p}(X \times Y)$ contains a complemented copy of $\mathbb{R}^{\omega} \approx C_{p}(\mathbb{N})$. If $X \times Y$ is pseudocompact, then $C_{p}(X \times Y)$ contains a complemented copy of $\left(c_{0}\right)_{p} \approx C_{p}([1, \omega])$, by [18, Theorem 1.4].

In view of Theorem 1.3 the following questions arise naturally.

Problem 2.9. Does the implication (1) $\rightarrow$ (2) in Theorem 1.3 remain valid without an assumption that $C_{p}(X)$ is distinguished?

Problem 2.10. Does the implication (3) $\rightarrow$ (2) in Theorem 1.3 remain valid without an assumption that $C_{p}(X)$ is distinguished? More specifically, does there exist a non-open continuous linear surjection $T: C_{p}[0,1] \rightarrow C_{p}[0,1]$ satisfying condition (3) of Theorem 1.3? 


\section{Distinguished spaces $C_{p}([1, \alpha])$ FOR COUntable ordinals $\alpha$}

In this section we are interested in finding a complete characterization of those spaces $Y$ which admit a continuous linear surjection $T: C_{p}(X) \rightarrow C_{p}(Y)$ for some simplest $\Delta$-spaces $X$.

1) Let $X$ be a discrete space. It follows from our [17, Corollary 3.3] that a continuous linear surjection $T: C_{p}(X) \rightarrow C_{p}(Y)$ exists if and only if $Y$ itself is discrete and $|Y| \leq|X|$.

2) Assume that $X$ is a metrizable compact space. Then, as we showed in [16, Proposition 3.5], $X$ is a $\Delta$-space if and only if $X$ is countable, equivalently, if and only if $X$ is homeomorphic to the ordinal space $[1, \alpha]$ for some countable ordinal $\alpha$, by the Mazurkiewicz-Sierpiński theorem. If we drop the requirement of linearity of the operator $T$, then for every metrizable compact space $Y$ there exists a continuous surjection $T: C_{p}([1, \omega]) \rightarrow C_{p}(Y)$, see [21, Proposition 5.4] and [19, Remark 3.4].

Let us fix an infinite countable ordinal $\alpha$ and ask for which ordinal $\beta \geq \alpha$ there exist a continuous linear isomorphism $T: C_{p}([1, \alpha]) \rightarrow C_{p}([1, \beta])$ ? The answer for this question has been given independently in [3] and [13].

Theorem 3.1. 3], [13] Let $\omega \leq \alpha \leq \beta<\omega_{1}$. Then the following conditions are equivalent:

(1) $C_{p}([1, \alpha])$ and $C_{p}([1, \beta])$ are isomorphic.

(2) The Banach spaces $C([1, \alpha])$ and $C([1, \beta])$ are isomorphic.

(3) $\beta<\alpha^{\omega}$.

Note that the most difficult implication $(2) \rightarrow(3)$ in the proof of Theorem 3.1 in both papers [3] and [13] is based on the result of C. Bessaga and A. Pełczyński [6, Lemma 2]. However, the proof of this key Lemma 2 presented in [6] heavily depends on the assumption that there exists a linear isomorphism $T: C([1, \alpha]) \rightarrow C([1, \beta])$ and it does not work if we assume only that $T$ is a continuous linear surjection even for $\alpha=\omega$. This obstacle has been resolved in the following new result.

Theorem 3.2. Let $\alpha$ be a fixed infinite countable ordinal. Then for a Tychonoff space $Y$ the following are equivalent.

(1) There exists a linear continuous surjection $T: C_{p}([1, \alpha]) \rightarrow C_{p}(Y)$.

(2) $Y$ is homeomorphic to $[1, \beta]$, where $\beta$ is a countable ordinal such that either $\beta<\alpha$, or $\alpha \leq \beta<\alpha^{\omega}$.

(3) $Y$ is homeomorphic to $[1, \beta]$, where $\beta$ is a countable ordinal with the property: if $\alpha \leq \beta$ then $C_{p}([1, \alpha])$ and $C_{p}([1, \beta])$ are isomorphic.

Proof. Implication $(2) \Rightarrow(3)$ is an immediate consequence of Theorem 3.1. If $\beta<\alpha$ then we define the operator of restriction: $T:\left.f \mapsto f\right|_{[1, \beta]}$. If $\alpha \leq \beta$, then 
the assumption of (3) says that there is an isomorphism between $C_{p}([1, \alpha])$ and $C_{p}([1, \beta])$ which trivially implies $(3) \Rightarrow(1)$. Now we prove $(1) \Rightarrow(2)$.

It is probably a folklore that (1) implies that $Y$ has to be a countable compact space. For instance, one can argue as follows. First, $Y$ is a metrizable compact space by [24, Theorem 2.7]. Second, $Y$ is scattered by [17, Proposition 3.9]. Every scattered metrizable compact space is homeomorphic to $[1, \beta]$ for some countable ordinal $\beta$. If $\beta<\alpha$ there is nothing to prove. So, let us assume that $\alpha \leq \beta$.

Applying the Closed Graph Theorem we consider $T$ as a linear bounded operator from the Banach space $C([1, \alpha])$ onto the Banach space $C([1, \beta])$. Note that in a particular case $\alpha=\omega$ we have that $C([1, \alpha]) \approx c_{0}$, hence $C([1, \beta])$ is a quotient of $c_{0}$, and we can apply the theorem of W.B. Johnson and M. Zippin [15] to deduce that the space $C([1, \beta])$ is isomorphic to $c_{0}$. By Theorem 3.1 we conclude that in this particular case $\beta<\omega^{\omega}$.

In a general case of arbitrary countable ordinal $\alpha$ we will apply more elaborate results of functional analysis. Recall that the Szlenk index of a Banach space $E$, denoted $\mathrm{Sz}(E)$, is an ordinal number, which is invariant under linear isomorphisms. For the definition of $\mathrm{Sz}(E)$ and its basic properties we refer the reader to the survey papers [23], [30]. The key tool is the following precise result of $\mathrm{C}$. Samuel, which is in turn based on a deep result of D. Alspach and Y. Benyamini [1], (see [23, Theorem 7], [30, Theorem 2.15]).

Fact A. For any $0 \leq \gamma<\omega_{1}$

$$
\mathrm{Sz}\left(C\left(\left[1, \omega^{\omega^{\gamma}}\right]\right)\right)=\omega^{\gamma+1} .
$$

We need also

Fact B. [30, Corollary 2.19] Let $E_{1}$ and $E_{2}$ be given Banach spaces with normseparable duals. Assume that $E_{2}$ is isomorphic to a subspace of a quotient space of $E_{1}$. Then $\mathrm{Sz}\left(E_{2}\right) \leq \mathrm{Sz}\left(E_{1}\right)$.

In order to finish the proof of $(1) \Rightarrow(2)$ suppose the contrary: $\beta \geq \alpha^{\omega}$. Then by Fact $\mathrm{A}, \mathrm{Sz}(C([1, \beta]))>\mathrm{Sz}(C([1, \alpha]))$ which contradicts Fact $\mathrm{B}$.

Remark 3.3. Linear continuous surjections $T: C_{p}([1, \alpha]) \rightarrow C_{p}([1, \beta])$ constitute only a proper part of the set of all bounded operators between corresponding Banach spaces. It would be very helpful to find a more direct topological argument for the proof of $\beta<\alpha^{\omega}$ in (1) $\Rightarrow(2)$ above.

An lcs $E$ is called hereditarily distinguished if every closed linear subspace of $E$ is distinguished [17]. Every Tychonoff product $\mathbb{R}^{X}$ is hereditarily distinguished. However, it is known that even a Fréchet distinguished lcs can contain a closed non-distinguished subspace. Problem 3.18 [17] asks: Does there exist an infinite compact space $X$ such that $C_{p}(X)$ is hereditarily distinguished? Below we answer in the affirmative to this question. 
Theorem 3.4. The space $C_{p}([1, \alpha])$ is hereditarily distinguished for every countable ordinal $\alpha$.

Proof. Denote $X=[1, \alpha]$. Then the product $\mathbb{R}^{X}$ is metrizable, since $X$ is countable. Let $E$ be any closed linear infinite-dimensional subspace of $C_{p}(X)$. If $f \in \mathbb{R}^{X}$ belongs to the closure of $E$ in $\mathbb{R}^{X}$, then there is a sequence $\left(f_{n}\right)$ in $E$ converging to $f$. This sequence provides a bounded set in $E$. This means that $E$ is a dense large subspace of $\mathbb{R}^{Y}$ for some infinite countable set $Y$. Then the strong duals of both spaces $E$ and $\mathbb{R}^{Y}$ are the same and carry the finest locally convex topology since the strong dual of $\mathbb{R}^{Y}$ is $\phi$.

\section{FROM POINTWISE TOPOLOGY TO WEAK TOPOLOGY}

M. Krupski proved that if $X$ is an infinite metrizable $C$-space then $C_{p}(X)$ and $C_{k}(X)_{w}$ are not homeomorphic [20]. Recall that a normal space $X$ is said to be a $C$-space if for any given sequence of its open covers $\left\{\mathcal{U}_{n}: n \in \omega\right\}$ there exists a sequence $\left\{\mathcal{V}_{n}: n \in \omega\right\}$ of families of pairwise disjoint open sets such that $\mathcal{V}_{n}$ is a refinement of $\mathcal{U}_{n}$ for each $n \in \omega$, and $\bigcup_{n \in \omega} \mathcal{V}_{n}$ is a cover of $X$.

Every countable-dimensional (in particular, every finite-dimensional space) is a $C$-space. It is well known that the Hilbert cube $Q=[0,1]^{\omega}$ is not a $C$-space.

Furthermore, M. Krupski and W. Marciszewski proved that if $K$ and $L$ are infinite compact spaces, then there is no a homeomorphism $\Phi: C_{k}(K)_{w} \rightarrow C_{p}(L)$, which is in addition uniform (see [22, Proposition 3.1]). In particular, a linear homeomorphism between $C_{p}(L)$ and $C_{k}(K)_{w}$ does not exist. Note that the proof presented in [22] uses very essentially the assumption that $\Phi^{-1}(A)$ is a compact subset of $C_{k}(Y)_{w}$ provided $A$ is a converging sequence in $C_{p}(L)$, and it is not clear whether this assumption can be removed. These remarks explain our motivation for the next open question.

Problem 4.1. Does there exist an infinite compact space $X$ admitting a continuous linear surjection $T: C_{p}(X) \rightarrow C_{k}(X)_{w}$ ?

In Theorem 4.4 we show that such $X$ (if exists) cannot be an infinite metrizable compact $C$-space. In particular, $X$ cannot be an infinite metrizable finitedimensional compact space, equivalently, an infinite compact subspace of the Euclidean space $\mathbb{R}^{n}$.

Observation 4.2. If $Y$ is an infinite Tychonoff space and $T: C_{p}(X) \rightarrow C_{k}(Y)_{w}$ is a continuous open linear surjection, then the topologies $\tau_{k}$ and $w$ coincide on $C(Y)$. Hence such an operator $T$ does not exist if $Y$ is an infinite compact space.

Proof. Indeed, let $\widehat{T}: C_{p}(X) / \operatorname{ker}(T) \rightarrow C_{k}(Y)_{w}$ be an isomorphism associated with $T$, where $\widehat{T} \circ P=T$ and $P: C_{p}(X) \rightarrow C_{p}(X) / \operatorname{ker}(T)$ is the quotient map. Since $C_{p}(X)$ is quasibarrelled (hence also $C_{p}(X) / \operatorname{ker}(T)$ ), the space $C_{k}(Y)_{w}$ is quasibarrelled. But the topology $\tau_{k}$ is $w$-polar, so $w=\tau_{k}$. 
Assuming that $X$ in the above Claim 4.2 is a $\Delta$-space one can remove a restriction that $T$ is open.

Theorem 4.3. Let $T: C_{p}(X) \rightarrow C_{k}(Y)_{w}$ be a continuous linear surjection, where $X$ is a $\Delta$-space and $Y$ is a Tychonoff space. Then $C_{k}(Y)_{w}=C_{p}(Y)=C_{k}(Y)$. Consequently, if $Y$ is an infinite compact space and $X$ is a $\Delta$-space, then such an operator $T$ does not exist.

Proof. Following the first part of the proof of $(1) \Rightarrow(2)$ in Theorem 2.2 the strong dual of $C_{k}(Y)_{\sigma}$ is free. Since $C_{k}(Y)$ and $C_{k}(Y)_{w}$ have the same bounded sets and the same topological dual, $C_{k}(Y)_{\beta}^{\prime}$ is free. By Theorem 2.2, we obtain that $\tau_{p}=\tau_{k}$ on $C(Y)$. This is impossible for infinite compact space $Y$.

Theorem 4.4. For an infinite metrizable compact $C$-space $X$ a continuous linear surjection $T: C_{p}(X) \rightarrow C_{k}(X)_{w}$ does not exist.

Proof. On the contrary, assume that such $X$ and $T$ exist. First we eliminate a possibility that $X$ is countable. Indeed, every countable $X$ is a $\Delta$-space and we can apply Theorem 4.3. Let $X$ be uncountable, then by the celebrated Milyutin theorem the Banach spaces $C_{k}(Q)$ and $C_{k}(X)$ are isomorphic, where $Q$ denotes the Hilbert cube, so also $C_{k}(Q)_{w}$ and $C_{k}(X)_{w}$ are isomorphic. Since the identity mapping from $C_{k}(Q)_{w}$ onto $C_{p}(Q)$ is continuous, we get a continuous linear surjection $S: C_{p}(X) \rightarrow C_{p}(Q)$. Our next argument is based on an analysis of the dual spaces $C_{p}(X)^{\prime}=L_{p}(X)$ (for more details see proofs of [19, Proposition 2.1] and [25, Theorem 3.4]).

The dual mapping $S^{*}$ embeds $L_{p}(Q)$ into $L_{p}(X)$, and therefore $Q$ is homeomorphic to a subspace of $L_{p}(X)$. For each natural $n \in \mathbb{N}$ consider the subspace $B_{n}(X)$ of $L_{p}(X)$ formed by all words of the reduced length $\leq n$ over $X$, and let $A_{n}(X)=B_{n}(X) \backslash B_{n-1}(X)$ be the subspace of all words of the reduced length $n$. It is known that each $B_{n}(X)$ is closed in $L_{p}(X)$, and $A_{n}(X)$ is homeomorphic to the subspace $\left(\mathbb{R}^{*}\right)^{n} \times\left(X^{n} \backslash \Lambda_{n}\right)$ of the Cartesian product $\left(\mathbb{R}^{*}\right)^{n} \times X^{n}$, where $\mathbb{R}^{*}=\mathbb{R} \backslash\{0\}$ and $\Lambda_{n}=\left\{\left(x_{1}, \ldots, x_{n}\right) \in X^{n}: x_{i}=x_{j}\right.$ for some $\left.i \neq j\right\}$. Obviously, $\Lambda_{n}$ is closed in the metrizable space $X^{n}$, hence $\Lambda_{n}$ is a $G_{\delta^{-}}$set in $X^{n}$ and $A_{n}(X)$ is a $F_{\sigma^{-}}$set in $\left(\mathbb{R}^{*}\right)^{n} \times X^{n}$. Each Cartesian product $\left(\mathbb{R}^{*}\right)^{n} \times X^{n}$ is a $C$-space by [29, Theorem 3], then each $A_{n}(X)$ is a $C$-space by [29, Subspace Theorem], hence $L_{p}(X)=\bigcup_{n \in \omega} A_{n}(X)$ is a $C$-space by [29, Sum Theorem] and finally, the Hilbert cube $Q$ would be also a $C$-space, which is false. The obtained contradiction finishes the proof of Theorem 4.4.

Problem 4.5. Find a characterization of metrizable compact spaces $X$ admitting a continuous linear surjection $T: C_{p}(X) \rightarrow C_{p}(Q)$.

Remark 4.6. R. Daverman kindly informed us that apparently there should exist a metrizable compact space $X$ with the following properties: the square $X^{2}$ is homeomorphic to the Hilbert cube $Q$, but $X$ itself does not contain a 
homeomorphic copy of $Q$. By these reasons a simple answer to Problem 4.5 seems to be unavailable. Obviously, $X$ cannot be a $C$-space, by the proof of Theorem 4.4. Note also that a continuous linear surjection $T: C_{p}[0,1] \rightarrow C_{p}(X)$ exists for any finite-dimensional metrizable compact space $X$ [24].

\section{REFERENCES}

[1] D. E. Alspach, Y. Benyamini, $C(K)$ quotients of separable $\mathcal{L}_{\infty}$-spaces, Israel J. Math. 32 1979, 145-160.

[2] A. V. Arkhangel'skiı̌, $C_{p}$-theory, in: Recent Progress in General Topology (M. Hušek and J. Van Mill, eds), Elsevier, Oxford, 1992, 1-56.

[3] J. Baars, J. de Groot, An isomorphical classification of function spaces of zero-dimensional locally compact separable metric spaces, Comment. Math. Univ. Carolinae 29 (1988), 577595.

[4] T. Banakh, J. Kąkol, W. Śliwa, Josefson-Nissenzweig property for $C_{p}$-spaces, RACSAM 113 (2019), 3015-3030.

[5] T. Banakh, J. Ka̧kol, J. Schürz, $\omega^{\omega}$-Base and infinite-dimensional compact sets in locally convex spaces, to appear in Revista Complutence.

[6] C. Bessaga, A. Pełczyński, Spaces of continuous functions (IV), Studia Math. 19 (1960), $53-62$.

[7] K. D. Bierstedt, J. Bonet, Some aspects of the modern theory of Fréchet spaces, RACSAM 97 (2003), 159-188.

[8] J. C. Ferrando, J. Ka̧kol, Metrizable bounded sets in $C(X)$ spaces and distinguished $C_{p}(X)$ spaces, J. Convex. Anal. 26 (2019), 1337-1346.

[9] J. C. Ferrando, J. Ka̧kol, A. Leiderman, S. A. Saxon, Distinguished $C_{p}(X)$ spaces, RACSAM (2021) 115:27, https://doi.org/10.1007/s13398-020-00967-4

[10] J. C. Ferrando, S. A. Saxon, If not distinguished, is $C_{p}(X)$ even close?, Proc. AMS 149 (2021), 2583-2596.

[11] A. Grothendieck, Sur les espaces $(F)$ et $(D F)$, Summa Brasil. Math. 3 (1954), 57-123.

[12] S. P. Gul'ko, The space $C_{p}(X)$ for countable infinite compact $X$ is uniformly homeomorphic to $c_{0}$, Bull. Acad. Pol. Sci. 36 (1988), 391-396.

[13] S. P. Gul'ko, Free topological groups and spaces of continuous functions on ordinals, Vestnik Tomsk State University (in Russian) 280 (2003), 34-38.

[14] H. Jarchow, Locally Convex Spaces, B.G. Teubner, Stuttgart, 1981.

[15] W. B. Johnson, M. Zippin, On subspaces of quotients of $\left(\sum G_{n}\right)_{\ell_{p}}$ and $\left(\sum G_{n}\right)_{c_{0}}$, Israel J. Math. 13 (1972), 311-316.

[16] J. Ka̧kol, A. Leiderman, A characterization of $X$ for which spaces $C_{p}(X)$ are distinguished and its applications, Proc. AMS, Ser. B, 8 (2021), 86-99.

[17] J. Ka̧kol, A. Leiderman, Basic properties of $X$ for which spaces $C_{p}(X)$ are distinguished, 2021, arXiv:2104.10506.

[18] J. Ka̧kol, D. Sobota, W. Marciszewski, L. Zdomskyy, On complemented copies of the space $c_{0}$ in spaces $C_{p}(X \times Y)$, https://arxiv.org/abs/2007.14723, to appear in Israel J. Math.

[19] K. Kawamura, A. Leiderman, Linear continuous surjections of $C_{p}$-spaces over compacta, Topology and Appl. 227 (2017), 135-145.

[20] M. Krupski, On the weak and pointwise topologies in function spaces, RACSAM 110 (2016), 557-563.

[21] M. Krupski, W. Marciszewski, A metrizable $X$ with $C_{p}(X)$ not homeomorphic to $C_{p}(X) \times$ $C_{p}(X)$, Israel J. Math. 214 (2016), 245-258. 
[22] M. Krupski, W. Marciszewski, On the weak and pointwise topologies in function spaces II, J. Math. Anal. Appl. 452 (2017), 646-658.

[23] G. Lancien, A survey on the Szlenk index and some of its applications, RACSAM 100 (2006), 209-235.

[24] A. Leiderman, S. Morris, V. Pestov, Free abelian topological groups and the free locally convex space on the unit interval, J. London Math. Soc. 56 (1997), 529-538.

[25] A. Leiderman, M. Levin, V. Pestov, On linear continuous open surjections of the spaces $C_{p}(X)$, Topology Appl. 81 (1997), 269-279.

[26] H. P. Lotz, N. T. Peck, H. Porta, Semi-embedding of Banach spaces, Proc. Edinburgh Math. Soc. 22 (1979), 233-240.

[27] P. Pérez Carreras and J. Bonet, Barrelled Locally Convex Spaces, North-Holland Mathematics Studies, 131, North-Holland, Amsterdam, 1987.

[28] G. M. Reed, On normality and countable paracompactness, Fund. Math. 110 (1980), 145152.

[29] D. M. Rohm, Products of infinite-dimensional spaces, Proc. AMS 108 (1990), 1019-1023.

[30] H. Rosenthal, The Banach space $C(K)$, in: Handbook of Geometry of Banach spaces vol. II, Chapter 36, (W. B. Johnson and J. Lindenstrauss, eds), Elsevier, 2003, 1547-1602.

Faculty of Mathematics and Informatics, A. Mickiewicz University, 61-614 Poznań, Poland and Institute of Mathematics Czech Academy of Sciences, Prague, CzeCh Republic

Email address: kakol@amu.edu.pl

Department of Mathematics, Ben-Gurion University of the Negev, Beer Sheva, P.O.B. 653, ISRAEL

Email address: arkady@math.bgu.ac.il 